\title{
Two-crystal, synchronously pumped, femtosecond optical parametric oscillator
}

\author{
V. Ramaiah-Badarla, ${ }^{1, *}$ A. Esteban-Martin, ${ }^{1}$ and M. Ebrahim-Zadeh ${ }^{1,2}$ \\ ${ }^{1}$ ICFO—Institut de Ciencies Fotoniques, Mediterranean Technology Park, 08860 Castelldefels, Barcelona, Spain \\ ${ }^{2}$ Institucio Catalana de Recerca i Estudis Avancats (ICREA), Passeig Lluis Companys 23, Barcelona 08010, Spain \\ *Corresponding author: venkata.badarla@icfo.es
}

Received November 10, 2014; revised December 12, 2014; accepted December 15, 2014;

posted December 16, 2014 (Doc. ID 226546); published January 20, 2015

\begin{abstract}
We demonstrate a femtosecond optical parametric oscillator based on two nonlinear crystals synchronously pumped by a single ultrafast laser for efficient intracavity signal amplification and output power enhancement. By deploying two identical MgO:PPLN crystals in a single standing-wave cavity, and two pump pulse trains of similar average power from the same Kerr-lens-mode-locked Ti:sapphire laser, a minimum enhancement of $56 \%$ in the extracted signal power is achieved, with un-optimized output coupling, when temporal synchronization between the two intracavity signal pulse trains is established, resulting in a corresponding enhancement of $49 \%$ in pump depletion. Using intracavity dispersion control, near-transform-limited signal pulses with clean spectrum are obtained. () 2015 Optical Society of America

OCIS codes: (190.7110) Ultrafast nonlinear optics; (190.4970) Parametric oscillators and amplifiers; (190.4400) Nonlinear optics, materials; (140.3600) Lasers, tunable.

http://dx.doi.org/10.1364/OL.40.000324
\end{abstract}

Synchronously pumped optical parametric oscillators (OPOs) have demonstrated their promise as versatile and indispensible sources of widely tunable femtosecond and picosecond pulses in spectral regions inaccessible to mode-locked lasers [1]. Over the past two decades, many such OPOs based on a variety of birefringent and quasiphase-matched nonlinear materials have been developed, enabling access to high-repetition-rate femtosecond pulses across extended spectral regions from the ultraviolet to the mid-infrared [2-9] and with durations down to a few optical cycles [10]. Traditionally, all such ultrafast OPOs deploy a single nonlinear crystal as the gain element within a cavity that is synchronously driven by a single input pump pulse train. In a departure from this conventional approach, we previously demonstrated that the inclusion of a second nonlinear crystal in an OPO cavity can offer additional advantages, such as enhanced wavelength flexibility, while maintaining robust and practical performance of the device with regard to all important operating parameters including output power, efficiency, power stability, as well as spectral and spatial beam quality [11-15]. We demonstrated successful operation of OPOs in both femtosecond [11] and picosecond [12] time-scale, as well as in continuous-wave (cw) regime [13-15], using two-crystal configurations, enabling dual-wavelength signal-idler pair generation with independent and arbitrary tuning across broad spectral regions. Dual-wavelength nanosecond OPOs have also been reported [16]. While in synchronously pumped femtosecond operation, we employed an antiresonant ring (ARR) interferometer to connect the two resonators [11], and in picosecond operation, we used a single optical cavity for the two-crystal OPO [12]. In the cw regime, we demonstrated the concept using both a single optical cavity $[\underline{13}, 14]$ and an ARR to couple the two OPOs [15]. While the primary goal of our earlier experiments on twocrystal OPOs has so far been arbitrary dual-wavelength generation, it would be desirable to also explore the feasibility of exploiting the concept to achieve further improvements in the OPO performance, for example, with regard to gain, efficiency, and output power enhancement. This can, in principle, be attained through intracavity optical parametric amplification (IOPA) of the circulating signal field in both crystals when operating at identical wavelengths, as observed in our earlier experiments on a two-crystal cw OPO in a single optical cavity $[\underline{13}, \underline{14}]$. In femtosecond OPOs, the exploitation of IOPA can be particularly advantageous for gain and efficiency enhancement because of the restrictions imposed by the spectral acceptance bandwidth (SAB) and group velocity mismatch (GVM) on the maximum usable interaction length of the nonlinear crystal for efficient parametric conversion. Given the relatively broad spectral bandwidth and short duration associated with femtosecond pump pulses, only crystals of short interaction length (typically $<1-2 \mathrm{~mm}$ ) can be utilized to minimize the effects of SAB and GVM. In critically phase-matched configurations, spatial walkoff is another factor restricting the useful interaction length, since the relatively low intensities associated with high-repetition-rate pump pulses demand tight focusing. On the other hand, by deploying multiple thin nonlinear crystals, one can circumvent such limitations and maintain the same SAB, GVM and spatial walkoff as that of a single crystal of short length [14], while increasing the total effective interaction length for parametric gain, thus enhancing the efficiency and output power through IOPA.

Here, we demonstrate this concept in a femtosecond OPO deploying a single optical cavity with two thin identical MgO:PPLN nonlinear crystals that are pumped in synchronization with two input pulse trains from the same Kerr-lens-mode-locked (KLM) Ti:sapphire laser. We show that such a scheme can provide major improvements in the performance of the femtosecond OPO with regard to conversion efficiency and output power. We achieve a minimum enhancement $\left(\left[\left(\mathrm{P}_{\mathrm{OPO}}\right.\right.\right.$ (sync) $\mathrm{P}_{\mathrm{OPO}}$ (unsync)) $/ \mathrm{P}_{\mathrm{OPO}}$ (unsync) $] \times 100$ ) of $56 \%$ in extracted signal power without output coupling optimization, enabling conversion of the pump to signal with enhanced efficiency, while avoiding the restrictions imposed by SAB 


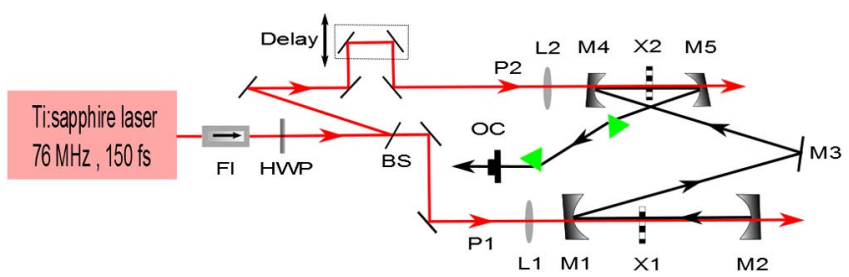

Fig. 1. Experimental setup for the two-crystal femtosecond OPO. FI, Faraday isolator; HWP, half-wave plate; BS, beam splitter; P1 and P2, pump pulse trains; L1 and L2, focusing lens; M1-M5, cavity mirrors; X1 and X2, MgO:PPLN crystals; OC, output coupler.

and GVM. It should be noted that while the use of multiple nonlinear crystals has been widely established in the context of external single-pass optical parametric generation and amplification of high-intensity pulses, this is to our knowledge the first demonstration of a multistep OPO/OPA involving low-energy femtosecond pulses, and in an intracavity configuration.

The schematic of the experimental setup for the twocrystal femtosecond OPO is shown in Fig. 1. The pump source is a KLM Ti:sapphire laser at $800 \mathrm{~nm}$ and $76 \mathrm{MHz}$ repetition rate. After transmission through an optical isolator and a half-wave plate for polarization control, the pump pulses have duration of $\sim 150 \mathrm{fs}$ with an average power of $850 \mathrm{~mW}$. The OPO is configured in a single standing-wave resonator with two intracavity focal planes, where the two nonlinear crystals (X1 and X2) are located. The resonator is formed by two pairs of identical concave mirrors (M1-M2, M4-M5) of radius of curvature, $r=10 \mathrm{~cm}$, a flat mirror (M3), and a $\sim 5 \%$ output coupler (OC) mounted on a translation stage to allow control of the cavity length for synchronization with the input pump pulse train. The OPO resonator is not symmetric with respect to the two focal planes, such that $\mathrm{X} 1$ is pumped as in a three-mirror asymmetric v-cavity with an additional focus at X2, while $\mathrm{X} 2$ is pumped as in a four-mirror symmetric x-cavity with an extra focus at X1. With the exception of the OC, all cavity mirrors are highly reflecting $(R>99 \%)$ for signal wavelengths over $1420-1580 \mathrm{~nm}$ and highly transmitting $(T>95 \%)$ for the pump at $800 \mathrm{~nm}$, with $>80 \%$ transmission for the idler over $3000-5000 \mathrm{~nm}$, hence ensuring singly resonant signal oscillation. The two nonlinear crystals are identical MgO:PPLN 500- $\mu \mathrm{m}$-long and 1-mm-thick, incorporating fanned gratings with periods from $\Lambda=16$ to $23 \mu \mathrm{m}$ across the 3.4-mm-wide aperture, both maintained at $100^{\circ} \mathrm{C}$. The crystal faces are antireflection (AR)-coated $(R<0.75 \%)$ for the signal and pump $(R<5 \%)$, with high transmission $(T>85 \%)$ for the idler over $1600-3500 \mathrm{~nm}$. Using a 50:50 thin-film beam-splitter (BS), the pump pulses are separated into two paths, $\mathrm{P} 1$ and $\mathrm{P} 2$, and directed to $\mathrm{X} 1$ and $\mathrm{X} 2$, respectively. Two lenses, L1 and L2 $(f=8 \mathrm{~cm})$, AR-coated $(R<1 \%)$ at $800 \mathrm{~nm}$, are then used to focus the beams to identical waist radii $\left(w_{0} \sim 16 \mu \mathrm{m}\right)$ inside the two crystals. The path, P2, includes a delay line so as to synchronize the pump pulse train inside the second crystal, X2. For group velocity dispersion (GVD) control, a pair of SF-11 prisms is deployed inside the cavity between M5 and the OC. The configuration of the two-crystal OPO used here offers a number of merits. The design ensures that both crystals use fresh pump beams, so that the input pump spectrum available to X2 is undepleted, and the same as that at the input to X1. The setup also allows independent control of input power, alignment, temporal synchronization, and focusing of the two pump beams in X1 and X2, to achieve optimum temporal overlap and spatial mode-matching with the intracavity signal in each crystal for maximum gain and efficiency enhancement.

To achieve successful operation of the two-crystal femtosecond $\mathrm{OPO}$, oscillation was first initiated with only one crystal, X1, in the cavity, pumped by $\mathrm{P} 1$ (with $\mathrm{P} 2$ blocked) at a signal wavelength of $1510 \mathrm{~nm}$ corresponding to a grating period of $\Lambda \sim 21.4 \mu \mathrm{m}$ in X1. Under this condition, oscillation was achieved at a pump power threshold of $200 \mathrm{~mW}$ (measured before L1), with $23.6 \mathrm{~mW}$ of signal power extracted through the OC for $425 \mathrm{~mW}$ of input pump power to X1. With the deployment of the second crystal, X2, inside the cavity, a small drop in the signal output power of $4 \mathrm{~mW}$ was observed, confirming the low insertion loss of the crystal coatings. The OPO oscillation was then re-initiated, but only pumping the crystal, X2, by P2 (with P1 blocked), and choosing the same signal wavelength of $1510 \mathrm{~nm}$ with a grating period of $\Lambda \sim 21.4 \mu \mathrm{m}$ for phase-matching in X2. In this case, the average pump threshold was $225 \mathrm{~mW}$ (measured before L2), with $22.5 \mathrm{~mW}$ of average signal power extracted through the OC for the same $425 \mathrm{~mW}$ of input pump power to X2. The small rise in threshold in this case was attributed to the slight increase in intracavity loss due to the presence of both crystals in the cavity, and small differences in mode-matching in $\mathrm{X} 1$ and $\mathrm{X} 2$ due to the asymmetric design of OPO resonator.

To study the output power characteristics of the twocrystal femtosecond OPO, we then measured the signal output power at different pumping levels. Figure 2(a) presents the data for the average signal power extracted through the $\sim 5 \%$ OC as a function of the input power when X1 and X2 are pumped individually with $50 \%$ of available power of $850 \mathrm{~mW}$. The input power in each case was measured before L1 and L2, respectively. The symmetric behavior of signal output power with only P1 or only P2 can be clearly seen from the plots. We then evaluated the OPO performance with both X1 and X2 in the

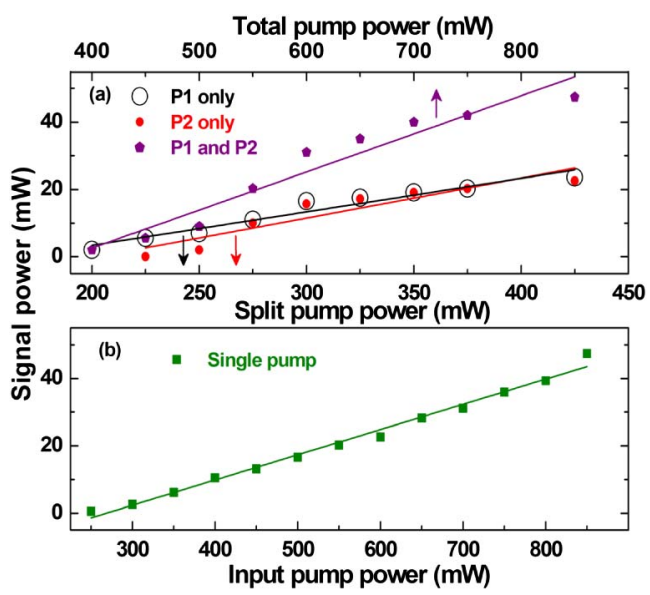

Fig. 2. Signal output power under different pumping conditions. (a) Split beams pumping X1, X2. (b) Single beam pumping $\mathrm{X} 1$. 
cavity, each pumped simultaneously with $50 \%$ of the available pump power, P1 and P2, and in the absence of synchronization between the two pump pulse trains in X2. This result is also shown in Fig. 2(a). The threshold pump power in this case was $400 \mathrm{~mW}$ (measured before BS), corresponding to $200 \mathrm{~mW}$ in P1 and P2 (measured before L1 and L2). In this configuration, we were able to extract a total signal power of $47.4 \mathrm{~mW}$ from the OPO for the maximum available input power of $850 \mathrm{~mW}$. This is close to the total power of $46.1 \mathrm{~mW}$ obtained with individual pumping of $\mathrm{X} 1$ and $\mathrm{X} 2$. Thus, the overall extracted signal power remained constant in both pumping configurations. We then compared the performance of the two-crystal OPO with the conventional single-crystal scheme by performing power scaling of the signal, but only pumping $\mathrm{X} 1$ with the full available pump power (in the absence of BS). The result is shown in Fig. 2 (b). In this case, a maximum signal power of $44 \mathrm{~mW}$ was extracted at the highest input power of $850 \mathrm{~mW}$. This value is nearly equal to the total signal power generated when both X1 and X2 are pumped simultaneously without synchronization, or individually, by the same total input pump power of $850 \mathrm{~mW}$. The OPO threshold in this case was $250 \mathrm{~mW}$ (measured before L1), which is nearly equal to the value when pumping X1 and X2 without synchronization.

In order to establish the merits of the two-crystal femtosecond OPO concept, the key parameter is the synchronization of the pump pulses with the resonant intracavity signal pulses in the two crystals. The data presented in Fig. 2(a) correspond to the case when there is no synchronization between $\mathrm{P} 1$ and $\mathrm{P} 2$, and hence the circulating signal pulses in X1 and X2 do not experience any additional gain. By adjusting the delay in the path of $\mathrm{P} 2$, the pump pulse train in X2 was thus synchronized to the circulating intracavity signal pulse train generated by $\mathrm{P} 1$ in $\mathrm{X} 1$. In this way, the signal pulses generated by X1 serve as seed in $\mathrm{X} 2$, and since $\mathrm{P} 1$ and $\mathrm{P} 2$ are now synchronized in time, intracavity amplification of the generated signal pulses can occur in X2. Similarly, the pump pulse train, $\mathrm{P} 1$, is synchronized in $\mathrm{X} 1$ with the signal pulse train generated by $\mathrm{P} 2$ in X2, and thus undergoes amplification in X1. As a result of this iterative OPO/IOPA process, a strong enhancement in parametric gain is experienced by the oscillating signal, resulting in an increase in the extracted power and efficiency.

To verify the enhancement in gain, we recorded the signal output power as a function of total input power with the two pump pulse trains, $\mathrm{P} 1$ and $\mathrm{P} 2$, in and out of synchronization. The result is shown in Fig. 3. When $\mathrm{P} 1$ and P2 are out of synchronization, the OPO signal power is nearly equal to the sum of the signal powers generated individually by each crystal. However, when P1 and P2 are synchronized, a strong enhancement in signal power is obtained with the maximum output increasing from 47.4 to $74 \mathrm{~mW}$ at the highest input power of $850 \mathrm{~mW}$ (measured before BS). The OPO signal power is now substantially higher than the sum of the signal powers generated individually by $\mathrm{P} 1$ and $\mathrm{P} 2$ without synchronization. The signal power enhancement varies from $1150 \%$ near threshold to $56 \%$ at $850 \mathrm{~mW}$ of pump power. It is also worthwhile to note that when pumping the two crystals in synchronization, the enhancement in signal power is

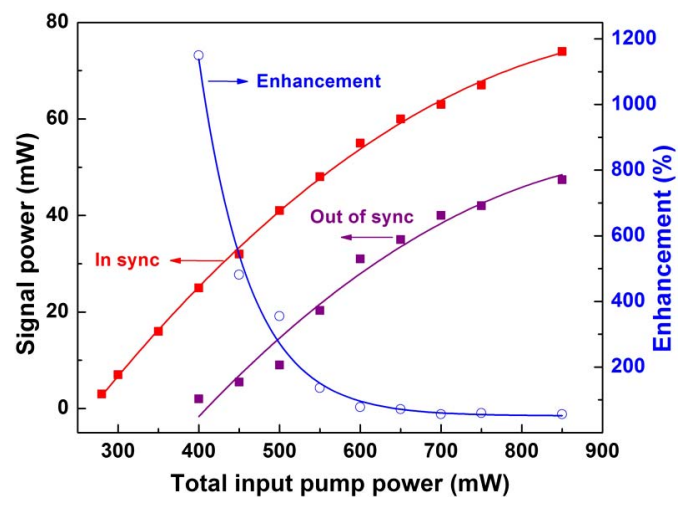

Fig. 3. Signal output power as a function of pump power with the input pulse trains to X1 and X2 in and out of synchronization, and the power enhancement.

$68 \%$ compared to pumping a single crystal with the total available input power of $850 \mathrm{~mW}$

We also recorded the variation of signal power with synchronization by adjusting the delay between the two pump pulse trains at $850 \mathrm{~mW}$ of pump power. As can be seen in Fig. 4, the maximum signal power with highest enhancement (56\%) is achieved at zero delay, as expected, when both pump pulse trains are exactly synchronized, with the signal power declining on either side of the zero delay. One can clearly see that beyond $45 \mu \mathrm{m}$ of delay, no enhancement in signal power is observed due to the lack of temporal overlap between the intracavity signal and input pump pulses inside X1 and X2. The enhancement in OPO performance was also confirmed by measurements of pump depletion with and without synchronization. In the absence of synchronization between $\mathrm{P} 1$ and $\mathrm{P} 2$, the maximum pump depletion was $61 \%$ at $850 \mathrm{~mW}$ of input power, whereas a significant increase of up to $91 \%$ was recorded under exact synchronization at zero delay. This represents an improvement of $49 \%$ in pump depletion.

We performed spectral and temporal characterization of output signal pulses from the two-crystal femtosecond OPO with and without intracavity GVD control. For spectral measurements, we used an optical spectrum analyzer, whilst temporal characterization was performed using interferometric autocorrelation based on twophoton absorption in a Si detector. The results are shown

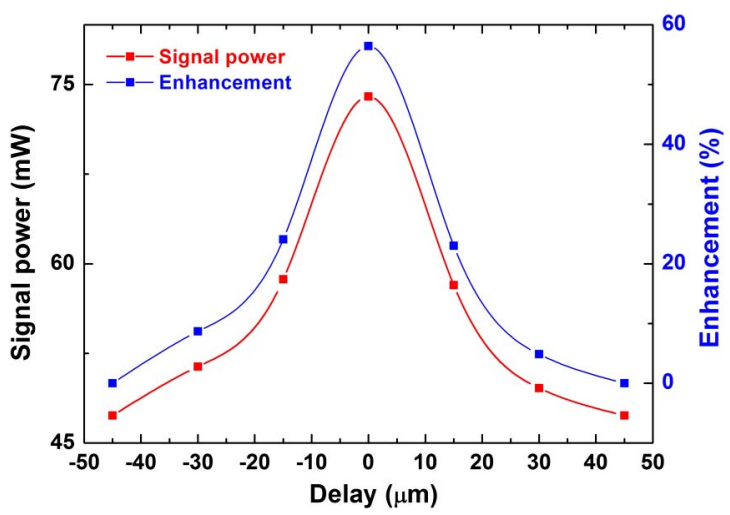

Fig. 4. Variation of signal power with respect to synchronization between $\mathrm{P} 2$ and $\mathrm{P} 1$, and the corresponding power enhancement. 

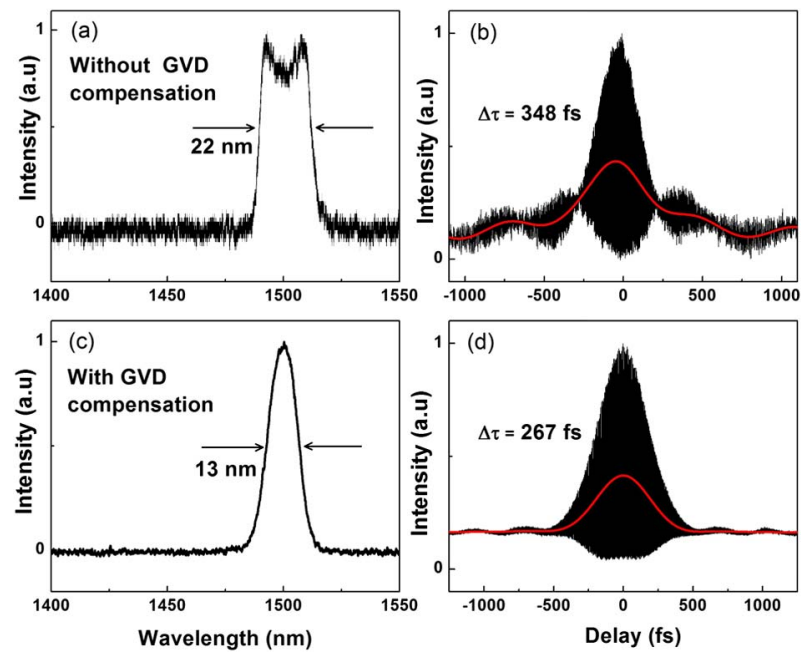

Fig. 5. Spectrum and interferometric autocorrelation of signal pulses. (a) and (b) without GVD control. (c) and (d) with GVD control.

in Fig. 5. As evident, in the absence of GVD compensation, the signal spectrum is double-peaked with a full width at half-maximum (FWHM) bandwidth of $22 \mathrm{~nm}$, and the pulses are strongly chirped with duration of 348 fs. Assuming sech ${ }^{2}$ temporal profile, the time-bandwidth product is $\Delta \nu \Delta \tau \sim 1.02$, which is nearly three times the transform limit $(\Delta \nu \Delta \tau \sim 0.32)$. With the inclusion of the SF11 prism pair in the cavity with a tip-to-tip separation of $15.4 \mathrm{~cm}$, we were able to obtain a smooth signal spectrum with a FWHM bandwidth of $13 \mathrm{~nm}$ and compress the pulses down to $267 \mathrm{fs}$, corresponding to a time-bandwidth product of $\Delta \nu \Delta \tau \sim 0.46$, close to transform limit. Therefore, the two-crystal femtosecond OPO can also provide high spectral and temporal characteristics.

In conclusion, we have demonstrated a synchronously pumped femtosecond OPO in a novel two-crystal design, which can provide substantial enhancement in nonlinear gain, efficiency, and output power through IOPA. The utilization of more than one nonlinear crystal in a single optical cavity allows major improvements in OPO performance by increasing the effective interaction length for gain, while avoiding the limitations of SAB and GVM. With nonoptimum output coupling, the technique has been shown to provide a minimum enhancement of $56 \%$ in the extracted power from the OPO and $49 \%$ in pump depletion, without compromising spectral and temporal performance of the output. With optimization of output coupling, further improvements in output power and extraction efficiency are expected. The generic technique can, in principle, also be extended to more than two nonlinear crystals in the cavity to achieve further enhancements in output power and efficiency through multistage IOPA. This can be particularly useful when low peak pump intensities and average powers may be involved. On the other hand, in the presence of high pumping intensities, the technique reduces the risk of optical damage or heating of the nonlinear material by distributing the optical and thermal load in more than one crystal, while increasing conversion efficiency and output power for the same total input pump power. The concept can also offer important advantages when deploying birefringent crystals under critical phasematching in the presence of spatial walkoff. By using crystals of short interaction length and independent focusing and mode-matching of the pump in each crystal, the effects of spatial walkoff can be circumvented, enabling operation of such OPOs in collinear phasematching configurations. These characteristics make the demonstrated concept a practical and universal approach to the development of efficient femtosecond OPOs in different spectral regions using ultrafast pump lasers of varying intensities and average powers. Moreover, since the technique is generic, it can be readily extended to other temporal domains, including picosecond and $\mathrm{cw}$ regime, and to other spectral regions.

We acknowledge support from the Ministry of Economy and Competitiveness (MINECO), Spain, through project OPTEX (TEC2012-37853), and the European Office of Aerospace Research and Development (EOARD) through grant FA8655-12-1-2128.

\section{References}

1. M. Ebrahim-Zadeh, in Solid-State Mid-Infrared Sources, I. T. Sorokina and K. L. Vodopyanov, eds. Topics in Applied Physics (Springer-Verlag, 2003), Vol. 89, pp. 179-218.

2. P. E. Powers, R. J. Ellingson, W. S. Pelouch, and C. L. Tang, J. Opt. Soc. Am. B 10, 2162 (1993).

3. D. T. Reid, G. T. Kennedy, A. Miller, W. Sibbett, and M. Ebrahimzadeh, J. Sel. Top. Quantum Electron. 4, 238 (1998).

4. M. Ghotbi, A. Esteban-Martin, and M. Ebrahim-Zadeh, Opt. Lett. 31, 3128 (2006).

5. S. T. Wong, T. Plettner, K. L. Vodopyanov, K. Urbanek, M. Digonnet, and R. L. Byer, Opt. Lett. 33, 1896 (2008).

6. A. Esteban-Martin, O. Kokabee, K. Moutzouris, and M. Ebrahim-Zadeh, Opt. Lett. 34, 428 (2009).

7. K. L. Vodopyanov, E. Sorokin, I. T. Sorokina, and P. G. Schunemann, Opt. Lett. 36, 2275 (2011).

8. A. Esteban-Martin, V. Ramaiah-Badarla, and M. EbrahimZadeh, Opt. Lett. 37, 1091 (2012).

9. V. Ramaiah-Badarla, A. Esteban-Martin, and M. EbrahimZadeh, Laser Photon. Rev. 7, L55 (2013).

10. S. Chaitanya Kumar, A. Esteban-Martin, T. Ideguchi, M. Yan, S. Holzner, T. W. Hänsch, N. Picqué, and M. Ebrahim-Zadeh, Laser Photon. Rev. 8, L86 (2014).

11. A. Esteban-Martin, V. Ramaiah-Badarla, and M. EbrahimZadeh, Laser Photon. Rev. 6, L7 (2012).

12. V. Ramaiah-Badarla, S. Chaitanya Kumar, and M. EbrahimZadeh, Opt. Lett. 39, 2739 (2014).

13. G. K. Samanta and M. Ebrahim-Zadeh, Opt. Lett. 36, 3033 (2011).

14. G. K. Samanta, A. Aadhi, and M. Ebrahim-Zadeh, Opt. Express 21, 9520 (2013).

15. K. Devi, S. Chaitanya Kumar, A. Esteban-Martin, and M. Ebrahim-Zadeh, Appl. Phys. B 114, 307 (2014).

16. T. Notake, K. Nawata, H. Kawamata, T. Matsukawa, F. Qi, and H. Minamide, Opt. Express 20, 25850 (2012). 